\title{
COMPARISON OF ENDOTRACHEAL INTUBATION VERSUS SUPRAGLOTTIC AIRWAY DEVICE IN PATIENTS UNDERGOING CORNEAL TRANSPLANTATION UNDER GENERAL ANAESTHESIA
}

\author{
Sana Abbas, Omar Zafar, Beenish Abbas, Basit Mehmood, Umer Hayat, Hassaan Javaid \\ Armed Forces Institute of Ophthalmology/National University of Medical Sciences (NUMS) Rawalpindi Pakistan
}

\begin{abstract}
Objective: To compare endotracheal intubation versus Suraglottic airway device in patients undergoing penetrating keratoplasty under general anaesthesia.

Study Design: Quasi-experimental study.

Place and Duration of Study: Armed Forces Institute of Ophthalmology, Rawalpindi, from Jan 2018 to Mar 2020.

Methodology: Total 28 patients enrolled with a mean age of $42.82 \pm 7.86$ years and age-range of 18-55 belonging to American Society of Anesthesiology I, II \& III undergoing penetrating keratoplasty under general anaesthesia. The airway was secured with I-Gel and Endotracheal tube in group A $(n=14)$ and group B $(n=14)$ respectively. Pre anaesthesia assessment along with informed written consent performed. General Anesthesia is given and patients monitored as per standard protocols. Postextubation incidence of cough, sore throat, bronchospasm, stridor and blood on device recorded in addition to surgery and recovery duration.

Results: Twenty-eight patients were enrolled in the study with a mean age of $42.82 \pm 7.86$ years. There were 9 (32.1\%) females and $19(67.9 \%)$ males in the study group. The recovery time was significantly lower for group A as compared to group B (72.64 \pm 5.06 vs $80.43 \pm 3.75, p<0.001)$. Similarly, a significantly lesser number of patients experienced post-extubation complications i.e. cough, among group A as compared to Group B [1 (7.1\%) vs 8 (57.1\%) p0.005].

Conclusion: I-Gel provides smooth recovery with reduced mean recovery time and decreased potential of raised intraocular pressure, when compared with endotracheal intubation in patients undergoing penetrating keratoplasty under general anaesthesia.
\end{abstract}

Keywords: Endotracheal intubation, Penetrating keratoplasty, Supraglottic airway device.

This is an Open Access article distributed under the terms of the Creative Commons Attribution License (https://creativecommons.org/licenses/by-nc/4.0/), which permits unrestricted use, distribution, and reproduction in any medium, provided the original work is properly cited.

\section{INTRODUCTION}

Corneal Transplant (Keratoplasty) is a common human organ donation, preliminary started in the $19^{\text {th }}$ century however, lack of anaesthetic availability led to unfavourable outcomes. Due to a wide variety of anaesthetic approaches, antibiotics and steroids, prosperous results can be achieved now a days ${ }^{1}$.

In 1940 Eye Banks were established, therefore shelf life of corneas increased up to 7-10 days from 2-3 days, due to improved storage conditions. Therefore, corneal transplantation can be performed as elective instead of urgent surgery nowadays ${ }^{2}$.

The cornea is a transparent structure lacking vascularity and act as a barrier. The main structural component is stroma and corneal endothelium, accountable for fluid transportation out of the eye. It is innervated by sympathetic nerve fibres and nasociliary branches of the ophthalmic division of trigeminal nerve ${ }^{3,4}$. Causes of corneal blindness are infective, congenital, degenerative, iatrogenic, trauma, vitamin A deficiency

Correspondence: Dr Sana Abbas, House No. 21, Street 14, Sector-F, DHA Phase II, Islamabad Pakistan

Received: 03 Apr 2020; revised received: 12 Jun 2010; accepted: 13 Jun 2020 and inflammatory responses (Steven Johnson Syndrome). Prevalence of corneal blindness in Pakistan is $1.8 \%{ }^{5}$. Indication of keratoplasty is endothelial disruption. There are several types of corneal transplant techniques. Penetrating Keratoplasty involving full-thickness corneal transplant. Selective Lamellar Keratoplasty, Anterior Lamellar Keratoplasty and Endothelial Keratoplasty, which involves only back layer corneal transplant. Advancements in corneal transplant involve Limbal epithelial stem cell transplantation and Keratoprosthesis (artificial cornea) 6,7.

Penetrating Keratoplasty technique routinely adopted at Armed Forces Institute of Ophthalmology for corneal transplantation, in which cornea sutured in place with either interrupted or continuous sutures. Typically, 16 sutures placed with four initial cardinal sutures placed at $12,3,6,9$ O'clock positions. Post-operative complications of keratoplasty are ocular content displacement, expulsive choroidal haemorrhage primary or late failure, glaucoma, endophthalmitis, corneal degenerations, mechanical trauma and wound leak ${ }^{8,9}$.

Anaesthetic techniques available are General Anesthesia, Regional Anesthesia and Topical Anes- 
thesia. Anaesthetic considerations for successful outcome in penetrating keratoplasty include good analgesia, maintenance of stable intraocular pressure and prevention of oculocardiac reflex throughout surgery. It is important to intercept post-extubation cough, nausea, vomiting and an increase in intraocular pressure as it can lead to suture damage ${ }^{10}$.

Our research was based on fact because endotracheal intubation increases intraocular pressure during induction and extubation due to cough and bucking on the endotracheal tube, therefore I-Gel can be safely used to secure airway in penetrating keratoplasty. Moreover, our secondary objective was to evaluate the effect on mean recovery time at extubation.

\section{METHODOLOGY}

This quasi-experimental study carried out at Armed Forces Institute of Ophthalmology, Rawalpindi from Jan 2018 to Mar 2020, approval taken from ethical research committee of the institute (218/ERC/AFIO). The sample size of 28 subjects (14 in each group) was calculated by WHO sample size calculator using a level of significance of $95 \%$, power of study $80 \%$, estimating incidence of post-extubation coughing complication of 5\% in an exposed group while 59\% in control group $^{11}$.

Corneas have limited shelf life of 6 days, therefore patients were prepared urgently upon arrival of corneas donated by the United States of America (USA). As per study protocol, all the patients interviewed, briefed, counselled about the procedure and informed written consent taken. Before reporting to the operation theatre, a detailed pre-anaesthesia assessment was carried out in all patients with necessary laboratory evaluation parameters to adhere with our inclusion and exclusion criteria, besides, to ensure patient safety which is of utmost concern in anaesthetic management.

All patients scheduled for penetrating keratoplasty surgery under general anaesthesia from both genders, age group between 18-55 years and American Society of Anesthesiologists status I, II or III, hemodynamically stable were included in the study. Nonconsenting patients, pregnant and lactating females, BMI >30, difficult airway, respiratory diseases and other contraindications to I-Gel were excluded.

Patients $(\mathrm{n}=28)$ divided into two groups, A-I Gel $(n=14)$ and B-endotracheal tube $(n=14)$. As preoperative preparation completion of essential documentation and an overnight fast/Nil per oral was ensu- red. On the day of surgery, patients brought to operation theatre and before initiating general anaesthesia standard monitoring such as blood pressure (non-invasive method), pulse oximeter ( $\mathrm{SpO} 2)$, end-tidal carbon dioxide (ETCO2) and electrocardiography electrodes attached.

18G IV cannula passed under aseptic conditions. Premedication performed with intravenous injections of nalbuphine $0.1 \mathrm{mg} / \mathrm{kg}$, paracetamol $15 \mathrm{mg} / \mathrm{kg}$, dexamethasone $0.08 \mathrm{mg} / \mathrm{kg}$ and metoclopramide $0.1 \mathrm{mg} /$ kg. Patients were Pre oxygenated with $100 \%$ oxygen for 3 minutes. Induction performed with intravenous propofol at a dose of $2 \mathrm{mg} / \mathrm{kg}$ and muscle relaxation achieved with $0.5 \mathrm{mg} / \mathrm{kg}$ of intravenous injection atracurium followed by laryngoscopy and intubation by a qualified anaesthetist 3 minutes later in case of endotracheal intubation. In case of I-Gel muscle relaxant wasn't given instead depth of anaesthesia achieved with inhalational anaesthetic (sevoflurane) before inserting I-gel. Inhalational anaesthetic isoflurane and injection atracurium $0.1 \mathrm{mg} / \mathrm{Kg}$ used for maintenance in the intubated group. Meticulous intraoperative monitoring was ensured, heart rate and mean arterial pressure below $20 \%$ was considered abnormal, respiratory rate and tidal volume were adjusted to achieve ETCO2 between 35-40 mmHg and SpO2 between $98-100 \%$. At the end of surgery, reversal was achieved with intravenous neostigmine \pm glycopyrrolate in endotracheal tube group whereas in I-Gel group reversal was not required. Average surgery duration recorded to be 5575 minutes in both groups.

Post-operative recovery time and mean surgical duration recorded. Post extubation complications cough, stridor, sore throat, bronchospasm and blood on device noted. Data entered and analyzed using SPSS-22. Quantitative variables including age, surgery duration and recovery time presented as mean and standard deviation. Qualitative variables including gender, American Society of Anesthesiologists Status, broncho-spasm, cough at extubation, blood on airway device presented in terms of frequency and percentages. Fischer-exact test used to compare categorical variables whereas; Mann-Whitney U-test used to compare continuous data. A $p$-value of $\leq 0.05$ considered statistically significant.

\section{RESULTS}

A total of 28 patients enrolled in the study with a mean age of $42.82 \pm 7.86$ years and age-range of 18-55. There were $9(32.1 \%)$ females and $19(67.9 \%)$ males in the study group. Among the study participants, 8 
(28.6\%) belonged to the American Society of Anesthesiology grade I, while 18 (64.3\%) and 2 (7.1\%) belonged to grade II and III respectively. Study participants were divided into two groups of 14 each; group A was exposed to I Gel whereas group B to the endotracheal tube. On comparing the groups in terms of surgery duration, no significant difference observed between group A and group B (63.21 \pm 6.07 vs $65.00 \pm 6.79, p-0.47)$. On comparing the recovery time among study groups, it was found that group A has significantly lower recovery time in hours as compared to group B (72.64 \pm 5.06 vs $80.43 \pm 3.75, p<0.001$ ) (table-I).

Among group A, significantly lesser number of patients experienced post-extubation complications as compared to group B in terms of cough i.e. 1 (7.1\%) vs $8(57.1 \%) p=0.005$. Whereas no significant differences were noted for other post-extubation complications including sore throat and blood on the device i.e. 1 $(7.1 \%)$ vs $2(14.3 \%), p=0.5$ and $1(7.1 \%)$ vs $0, p=0.1$ respectively as shown in table-II.

Table-I: Comparison of surgery duration and recovery time among the study groups.

\begin{tabular}{|c|c|c|c|}
\hline & \multicolumn{2}{|c|}{ Study Participants } & \multirow[b]{2}{*}{$\begin{array}{c}p- \\
\text { value }\end{array}$} \\
\hline & $\begin{array}{c}\text { Group A } \\
(n=14)\end{array}$ & $\begin{array}{c}\text { Group B } \\
(n=14)\end{array}$ & \\
\hline $\begin{array}{l}\text { Surgery duration in } \\
\text { minutes (mean } \pm \text { SD) }\end{array}$ & $\begin{array}{c}63.21 \pm \\
6.07 \\
\end{array}$ & $\begin{array}{c}65.00 \pm \\
6.79 \\
\end{array}$ & 0.47 \\
\hline $\begin{array}{l}\text { Recovery time in } \\
\text { minutes (mean } \pm S D)\end{array}$ & $\begin{array}{c}72.64 \pm \\
5.06\end{array}$ & $\begin{array}{l}80.43 \pm \\
3.75\end{array}$ & $<0.001^{*}$ \\
\hline
\end{tabular}

*Significant $p$-value; was calculated by applying Mann-Whitney U test Table-II: Comparison of post-extubation complications among the study groups.

\begin{tabular}{|c|c|c|c|}
\hline & \multicolumn{2}{|c|}{ Study Participants } & \multirow[b]{2}{*}{$\begin{array}{c}p- \\
\text { value }\end{array}$} \\
\hline & $\begin{array}{c}\text { Group A } \\
(n=14)\end{array}$ & $\begin{array}{c}\text { Group B } \\
(\mathrm{n}=14)\end{array}$ & \\
\hline Sore throat in recovery & $1(7.1 \%)$ & $2(14.3 \%)$ & 0.500 \\
\hline Cough at extubation & $1(7.1 \%)$ & $8(57.1 \%)$ & $0.005^{*}$ \\
\hline Blood on device & $1(7.1 \%)$ & - & 1.000 \\
\hline
\end{tabular}

Only one patient from group B, experienced postextubation bronchospasm and one patient experienced post-extubation stridor, while none of the patients from group A experienced any such conditions, and the differences were not significantly different $(p=0.1$ and $p=0.12$ respectively).

\section{DISCUSSION}

Data analysis of research interpreted statistically significant reduction of incidence of cough in I-Gel group, a common adverse effect encountered after endotracheal extubation leading to mean an increase in intraocular pressure which can damage sutures in penetrating keratoplasty. Moreover provided added benefit of reduction in recovery duration. However, IGel can cause minor local trauma expressed as blood on the device but when compared with advantages, this implication can be overlooked.

Guerrier et al performed randomized controlled trial to compare supraglottic airway device I-Gel and an endotracheal intubation in penetrating and lamellar keratoplasty performed under general anaesthesia. They concluded that the use of I-Gel reduces the risk of ocular hypertension and reduces recovery time, findings consistent with our study.

Recovery time was 75-86 minutes in I-Gel group whereas in endotracheal tube it was 82-95 minutes $(p=0.02)$, comparable to recovery time $72.64 \pm 5.06 \mathrm{mi}-$ nutes and $80.43 \pm 3.75$ minutes in I-Gel and endotracheal tube group respectively $(p<0.001)$ in this study. Statistically significant difference of cough at extubation was observed in endotracheal tube group ( $p$ $<0.001)$ consistent with our results $(p=0.005)$. There wasn't any considerable difference in stridor and sore throat between two groups ${ }^{11}$.

Panner et al compared I-gel versus endotracheal tube in patients undergoing elective caesarean section in a prospective randomized controlled trial to observe hemodynamic response at insertion and extubation. They observed that approximately $20 \%$ rise in mean arterial pressure and heart rate seen in endotracheal group when compared with I-Gel group $(p<0.001)$ thus providing greater hemodynamic stability. They concluded remarkable sore throat after endotracheal intubation $(p<0.001)$, however in our study, no obvious incidence of sore throat in recovery was seen in endotracheal group $(p=0.500)$. They compared both groups in terms of postoperative complication of blood on the device, which was not conspicuous in either of the groups consistent with findings of our study ${ }^{12}$.

Ziyaeifard et al, compared intraocular pressure and hemodynamic responses to the insertion of laryngeal mask airway or endotracheal tube using general anaesthesia with propofol and remifentanil in cataract surgery. They did not observe any significant difference in heart rate, systolic \& diastolic pressure and intraocular pressure, but laryngeal mask airway insertion was preferred due to ease of insertion and less trauma when compared with an endotracheal tube, therefore, preferred in cataract surgery. Although statistically insignificant $(p=1.00)$ but it is contradictory to our analysis as I-Gel causes more trauma when compared with endotracheal intubation ${ }^{13}$. 
Ismail et al, determined intraocular pressure and haemodynamic responses to insertion of the I-Gel, laryngeal mask airway or endotracheal tube in nonophthalmological surgeries. We suspected that endotracheal intubation leads to increase in intraocular pressure due to incidence of cough $(p=0.005)$ therefore their findings that intraocular pressure was significantly raised after insertion of the endotracheal tube (11.6 \pm 1.6 to $16.5 \pm 1.7 \mathrm{mmHg}$ ) when compared with Igel $(p<0.001)$ supports our suspicion ${ }^{14}$.

Luce et al, compared supraglottic airway devices versus endotracheal intubation in children in terms of respiratory complications such as laryngospasm, cough and breath-holding. They elaborated that the incidence of laryngospasm $(p=0.93)$ and cough $(p=0.41)$ were lower in the laryngeal mask airway group, therefore compatible with our results. Post-operative incidences of sore throat $(p=0.57)$, bronchospasm $(p=0.81)$ and blood staining on the device $(p=0.09)$ were homogenous therefore parallel with our results of sore throat $(p=0.5)$, bronchospasm $(p=0.1)$ and blood staining on the device $(p=1.0)^{15}$.

Danny et al, in their study reported a significantly higher incidence of cough at extubation and after extubation in endotracheal group in comparison with laryngeal mask airway group $(p<0.001)$, relevant to our study results $(p=0.005)^{16}$.

Saeki et el evaluated sore throat in respect of pain at rest score (0: no pain, 1: mild discomfort, 2: mild pain, 3: severe pain) in endotracheal intubation, laryngeal mask airway and cuffed oropharyngeal airway. Ten patients, 3 patients and 5 patients reported pain at rest (scores 1, 2, 3) in endotracheal, laryngeal mask airway and cuffed oropharyngeal group respectively. Therefore exhibiting greater incidence of sore throat in endotracheal group analogous to our study ${ }^{17}$.

Hamdan et al, compared post-operative vocal changes in patients using laryngeal mask airway and endotracheal intubation, no notable difference in the sore throat was seen between groups $(p=0.2)$, relatable to our results in respect to sore throat $(p=0.5)^{18}$.

Therefore, significant findings of our study are that I-Gel in contrast to endotracheal intubation provides better anaesthetic considerations in penetrating keratoplasty concerning postoperative complications. Moreover, it reduces recovery time therefore facilitating in busy surgical schedule in operation theatre. Although endotracheal intubation is the oldest and standard method of securing airway but newer trends in anaesthesia can be adopted weighing risk-benefit ratio to pace up with evolutions in the surgical field.

\section{CONCLUSION}

I-Gel provides smooth recovery with reduced mean recovery time and decreased potential of raised intraocular pressure, when compared with endotracheal intubation in patients undergoing penetrating keratoplasty under general anaesthesia.

\section{CONFLICT OF INTEREST}

This study has no conflict of interest to be declared by any author.

\section{REFERENCES}

1. Chua AWY, Chua MJ, Kam PCA. Recent advances and anaesthetic considerations in corneal transplantation. Anaesth Intens Care 2018; 46(2): 162-70.

2. Mannan R, Sharma N, Pruthi A, Maharana PK, Vajpayee RB. Penetrating keratoplasty for perforated corneal ulcers under topical anaesthesia. Cornea 2013; 32(11): 1428-31.

3. Yao WY, Li SY, Yuan YJ, Tan HS, Han NR, Sultana R, et al. Comparison of Supreme laryngeal mask airway versus endotracheal intubation for airway management during general anaesthesia for cesarean section: a randomized controlled trial. BMC Anesth 2019; 19(1): 123-28.

4. Cedrone C, Culasso F, Cesareo M, Nucci C. Incidence of blindness and low vision in a sample population: Priverno Eye Study, Italy. Ophthalmol 2003; 110(9): 584-88.

5. Jadoon MZ, Dineen B, Bourne RRA, Shah SP, Khan MA, Jhonson GJ. Blindness and visual impairment in Pakistan: The Pakistan National Blindness and visual impairment survey. Invest Ophthalmol Visual Sci 2006; 47(11): 4749-55.

6. Pant D, Koul A, Sharma B, Sood J. A comparative study of Laryngeal Mask Airway size 1 vs. i-gel size 1 in infants undergoing daycare procedures. Paediatr Anaesth 2015; 25(4): 386-91.

7. Jain S, Nazir N, Khan RM, Ahmed SM. A prospective randomized control study comparing classic laryngeal mask airway with Guedel's airway for tracheal tube exchange and smooth extubation. Anesth Essays Res 2016; 10(3): 552-56.

8. Modern H, Moshiri E, Yazdi B, Mohammadbeigi A, Modir A. Comparing the efficacy and safety of laryngeal mask airway, streamlined liner of the pharyngeal airway and I-gel following tracheal extubation. Med Gas Res 2018; 7(4): 241-46.

9. Sanket B, Ramavakoda CY, Nishtala MR, Ravishankar CK, Ganigara A. Comparison of second-generation supraglottic airway devices (i-gel versus LMA ProSeal) during elective surgery in Children. Aana J 2015; 83(4): 275-80.

10. Toman H, Erbas M, Sahin H, Kiraz HA, Uzun M, Ovali MA. Comparison of the effects of various airway devices on hemodynamic response and QTc interval in rabbits under general anaesthesia. J Clin Monit Comput 2015; 29(6): 727-32.

11. Guerrier G, Boutboul D, Rodent S, Hallal D, Levy J, Sjogren L, et al. Comparison of a Supraglottic Gel Device and an Endotracheal Tube in Keratoplasty Performed Under General Anesthesia: A Randomized Clinical Trial. Cornea 2016; 35(1): 37-40.

12. Panneer M. Comparison of I-gel versus endotracheal tube in patients undergoing elective cesarean section: a prospective randomized control study. Anesth Essays Res 2017; 11(4): 930-33.

13. Ziyaeifard M, Azarfarin R, Massoumi G. A comparison of intraocular pressure and hemodynamic responses to insertion of laryngeal mask airway or endotracheal tube using anaesthesia 
with propofol and remifentanil in cataract surgery. J Res Med Sci 2012; 17(6): 503-07.

14. Ismail SA, Bisher NA, Kandil HW, Mowafi HA, Atawia HA. Intraocular pressure and haemodynamic responses to insertion of the i-Gel, laryngeal mask airway or endotracheal tube. Eur J Anaesthesiol 2011; 28(6): 443-48.

15. Luce V, Harkouk H, Brasher C, Michelet D, Hilly J, Maesani M, et al. Supraglottic airway devices vs tracheal intubation in children: a quantitative meta-analysis of respiratory complications. Paediatr Anaesth 2014; 24(10): 1088-98.
16. Denny NM, Gadelrab R. Complications following general anaesthesia for cataract surgery: a comparison of the laryngeal mask airway with tracheal intubation. J R Soc Med 1993; 86(9): 521-22.

17. Saeki H, Morimoto Y, Yamashita A, Nagusa Y, Shimizu K, Oka $\mathrm{H}$, et al. Postoperative sore throat and intracuff pressure: comparison among endotracheal intubation, laryngeal mask airway and cuffed oropharyngeal airway. Masui 1999; 48(12): 1328-31.

18. Hamdan AL, Kanazi G, Rameh C, Rifai H. Immediate postoperative vocal changes in patients using laryngeal mask airway versus endotracheal tube. J Laryngol Otol 2008; 122(8): 829-35. 